\title{
Gender differences in the association of mixed anxiety and depression with suicide
}

Ottar Bjerkeset, Pål Romundstad and David Gunnell

\section{Summary}

The incidence of depression is higher in women than men but the reverse pattern is seen with suicide. In a cohort of 50692 Norwegians we found that suicide risk associated with comorbid anxiety and depression was two-fold higher in men $(\mathrm{OR}=7.4,95 \% \mathrm{Cl} 3.1-17.5)$ than women $(\mathrm{OR}=2.9$, 95\% C 0.8-10.6), although statistical evidence for a difference was weak $(P=0.4)$. If real, these gender differences could reflect either a more severe symptom profile in men with self-reported anxiety and depression, perhaps because of gender differences with regard to the stigma associated with mental illness, or gender differences in the way men respond to mental illness (e.g. self-medication/help-seeking).

\section{Declaration of interest}

None.
Depression is thought to be the most important antecedent of suicide; however, the epidemiology of suicide and depression differ in a number of respects. Most strikingly, in high-income countries, rates of suicide are three to four times higher in men than women. In contrast, population surveys indicate that the prevalence of depression, ${ }^{1}$ suicidal thoughts and suicide attempts ${ }^{2}$ is higher in women than men. There are several possible explanations for these contrasting patterns. ${ }^{3}$ First, men use more lethal methods of suicide than women, so case fatality is higher in men. Second, women with depression are more likely than men to seek help from friends, family and health services. ${ }^{4}$ Last, it is possible that depression is more stigmatised in men than in women, so men may be less likely to report symptoms. Evidence for this latter explanation is mixed. ${ }^{5,6}$ In a cohort study examining the association of psychiatric caseness (measured using the General Health Questionnaire (GHQ)) with suicide, GHQ-positive men were more than four times more likely to die by suicide than GHQ-positive women. This study was underpowered as only 16 suicides occurred over the follow-up period. It is noteworthy that in a Danish registerbased study, women who were admitted to hospital for the treatment of psychiatric illness appeared to be at greater suicide risk than men.

We have further investigated this issue in a large populationbased cohort of men and women from the Nord-Trøndelag region of Norway who completed the Hospital Anxiety and Depression Scale (HADS) ${ }^{8}$ questionnaire in 1995-1997. A previous analysis of this cohort ${ }^{9}$ found that mixed anxiety and depression was strongly associated $(\mathrm{OR} \geqslant 6.00)$ with suicide risk.

\section{Method}

The Nord-Trøndelag Health Study (HUNT 2) was carried out in 1995-1997 (www.hunt.ntnu.no/index.php?side=english). Participants completed the 14-item HADS. ${ }^{8}$ Of the 92936 eligible individuals aged 20 years and older, $66140(71.2 \%)$ entered HUNT 2. Of these, $50692(76.6 \%)$ had valid responses on all background variables, including depression (HADS-D) and anxiety (HADS-A) scores. The death registry (Statistics Norway; www.ssb.no/english) was used to identify all deaths up to 31 December 2004. Suicides were defined as deaths coded E950-E959 (suicide) and E980-E989 (excluding E988.8, undetermined intent) using ICD-9 or X60-84 (suicide) and Y10-34 (undetermined intent) using ICD-10.

We used Cox proportional hazards regression with calendar time as the time axis in Stata Version 9.0 for Windows to examine associations of mixed anxiety and depression with suicide. Mixed anxiety and depression was evaluated as a dichotomous variable using a cut-off score of $\geqslant 8$ on both HADS-A and HADS-D for caseness, ${ }^{10}$ and as a continuous variable using the total sum of HADS-A and HADS-D (HADS-T score). To study whether the associations differed in men compared with women, we fitted appropriate interaction terms.

Our initial models controlled for age (in 10-year bands) and gender. We then examined the effect on associations of controlling for marital/cohabitation status, frequency of alcohol consumption ( $\leqslant$ monthly, $2-4$ times per month, $\geqslant 5$ per month, $\leqslant$ monthly but previous alcohol problems), smoking (never, former, current), and educational level ( $<9$ years, 9-12 years, $>12$ years).

\section{Results}

Altogether 26044 women (mean age 46.5 years) and 24648 men (mean age 47.3) were included in the analysis. At baseline, 1542 $(5.9 \%)$ women and $1183(4.8 \%)$ men reached caseness for mixed anxiety and depression. More men than women reported alcohol intake $\geqslant 5$ times a month $(18.4 \% v .8 .1 \%, P<0.0001)$, but fewer men were daily smokers $(28.5 \%$ v. $30.7 \%, P<0.0001)$. Gender differences in other baseline characteristics were minor: $40.2 \%$ of women $v$. 38.8\% of men lived alone and $69.8 \%$ of women $v$. $71.5 \%$ of men had received $\geqslant 9$ years of education.

At the end of the follow-up period, $14(0.05 \%)$ women and 27 $(0.11 \%)$ men had died by suicide. Only 11 (27\%) suicides occurred among people with mixed anxiety and depression at baseline. Among the 30 remaining suicides, 18 had a HADS-D score below 4, whereas 12 scored 4-7. Of note there were no suicides among participants with 'pure' depression, i.e. HADS-D score $>8$ and HADS-A score $<8 \quad(n=2178)$.

In analyses controlling for age and gender, mixed anxiety and depression was strongly associated with suicide risk in an analysis based on men and women combined (HR=7.07, 95\% CI 3.514.25); this association was attenuated in the fully adjusted model ( $\mathrm{HR}=4.82,95 \%$ CI 2.43-9.55). In gender-stratified analyses the fully adjusted HR for suicide in men with mixed anxiety and 


\begin{tabular}{|c|c|c|c|c|c|c|}
\hline & \multicolumn{3}{|c|}{ Age-adjusted associations } & \multicolumn{3}{|c|}{ Fully adjusted associations ${ }^{a}$} \\
\hline & Women & Men & $P$ & Women & Men & $P$ \\
\hline \multicolumn{7}{|l|}{ HADS mixed anxiety and depression } \\
\hline Non-cases & 1.00 & 1.00 & & 1.00 & 1.00 & \\
\hline Cases & 4.13 & 9.20 & & 2.90 & 7.41 & \\
\hline $95 \% \mathrm{Cl}$ & $1.14-14.95$ & $3.97-21.30$ & 0.41 & $0.79-10.59$ & $3.14-17.51$ & 0.40 \\
\hline Hazard ratio per unit increase in HADS-T score & 1.08 & 1.13 & & 1.06 & 1.12 & \\
\hline $95 \% \mathrm{Cl}$ & $1.01-1.16$ & $1.08-1.18$ & 0.46 & $0.99-1.14$ & $1.06-1.17$ & 0.43 \\
\hline
\end{tabular}

depression at baseline was 7.41 (95\% CI 3.14-17.51) and in women it was 2.90 (95\% CI 0.79-10.59). Although the HR was over twofold higher in men than in women, there was no statistical evidence of a difference in gender-specific associations (Table 1).

\section{Discussion}

Caseness on a brief psychiatric rating scale was associated with a five-fold increased risk of suicide over an 8-year follow-up. In keeping with a previous study ${ }^{6}$ the effect estimates were indicative of higher suicide risk in men than women with mixed anxiety and depression but there was no statistical evidence to support such a difference. Both studies are relatively small and sizeable gender differences cannot be ruled out. Disparities in the size of the gender difference between the two studies could be attributed either to chance or to psychometric differences between the instruments used to measure mental disorder. Alhough the correlation between HADS-T and the GHQ is as high as $0.75,{ }^{10}$ they measure different symptom profiles. For example, anhedonia, the major depressive feature assessed by HADS, has been proven to be the least gender-specific among depressive symptoms. ${ }^{11}$

Surprisingly, most suicides occurred among HADS-negative participants and half of all suicides had HAD-D scores $<4$ at baseline, indicating either underreporting or, more plausibly, the relatively transient nature of common mental disorder.

There are several limitations to our study. First, all participant characteristics were measured only once and we had no information on life-events or anxiety and depression symptom fluctuation in the follow-up period. Second, although HADS can indicate caseness in a variety of settings ${ }^{10}$ it does not reflect the prevalence of more severe mental disorders known to increase suicide risk. Nevertheless, there is growing evidence that selfreported symptoms of anxiety and depression have good prognostic and predictive value. ${ }^{11,12}$ Last, although depression/ anxiety disorders account for over half of all suicides and are more common in women in the general population, other psychiatric conditions associated with suicide such as alcohol and drug misuse occur more frequently in men and may contribute to the gender disparities in suicide rates.

In summary, these findings provide some support for the notion that gender differences related to the risk of depressive disorders and suicide are attributable to gender-differences in the reporting of psychiatric symptoms. ${ }^{13}$ Other contributory factors may include gender differences in help-seeking, acceptable suicide methods, depressive symptom profile and the psychiatric conditions contributing to population suicide rates.
Ottar Bjerkeset, MD, PhD, Department of Research and Development, Levange Hospital, Nord-Trøndelag Health Trust, and HUNT Research Centre, Department of Neuroscience, Faculty of Medicine, Norwegian University of Science and Technology, Trondheim, Norway; Pål Romundstad, PhD, Department of Public Health and General Practice, Norwegian University of Science and Technology, Trondheim; David Gunnell, PhD, Department of Social Medicine, University of Bristol, UK

Correspondence: Ottar Bjerkeset, MD, PhD, Department for Research and Development, Levanger Hospital, Nord-Trøndelag Health Trust, Kirkegt. 27600 Levanger, Norway. Email ottar.bjerkeset@ntnu.no

First received 21 Sep 2007, final revision 4 Feb 2008, accepted 25 Feb 2008

\section{References}

1 Kringlen E, Torgersen S, Cramer V. A Norwegian psychiatric epidemiological study. Am J Psychiatry 2001; 158: 1091-8.

2 Bernal M, Haro JM, Bernert S, Brugha $T$, de Graaf R, Bruffaerts R, Lépine JP, de Girolamo G, Vilagut G, Gasquet I, Torres JV, Kovess V, Heider D, Neeleman $\mathrm{J}$, Kessler R, Alonso J. Risk factors for suicidality in Europe: results from the ESEMED study. J Affect Disord 2007; 101: 27-34.

3 Hawton K. Sex and suicide. Gender differences in suicidal behaviour. $\mathrm{Br} J$ Psychiatry 2000; 177: 484-5.

4 Biddle L, Gunnell D, Sharp D, Donovan JL. Factors influencing help seeking in mentally distressed young adults: a cross-sectional survey. Br J Gen Pract 2004; 54: 248-53.

5 Bogner HR, Gallo JJ. Are higher rates of depression in women accounted for by differential symptom reporting? Soc Psychiatry Psychiatr Epidemiol 2004; 39: 126-32.

6 Gunnell D, Rasul F, Stansfeld SA, Hart CL, Davey SG. Gender differences in self-reported minor mental disorder and its association with suicide. A 20year follow-up of the Renfrew and Paisley cohort. Soc Psychiatry Psychiatr Epidemiol 2002; 37: 457-9.

7 Qin P, Agerbo E, Westergård-Nielsen N, Eriksson T, Mortensen PB. Gender differences in risk factors for suicide in Denmark. Br J Psychiatry 2000; 177: $546-50$

8 Snaith RP, Zigmond AS. The hospital anxiety and depression scale. $\mathrm{Br}$ Med J (Clin Res Ed) 1986; 292: 344

9 Mykletun A, Bjerkeset O, Dewey M, Prince M, Overland S, Stewart R. Anxiety, depression, and cause-specific mortality: the HUNT study. Psychosom Med 2007; 69: 323-31.

10 Bjelland I, Dahl AA, Haug TT, Neckelmann D. The validity of the Hospital Anxiety and Depression Scale. An updated literature review. J Psychosom Res 2002; 52: 69-77.

11 Chen LS, Eaton WW, Gallo JJ, Nestadt G. Understanding the heterogeneity of depression through the triad of symptoms, course and risk factors: a longitudinal, population-based study. J Affect Disord 2000; 59: 1-11.

12 Paykel ES. Remission and residual symptomatology in major depression. Psychopathology 1998; 31: 5-14.

13 Blair-West GW, Mellsop GW. Major depression: does a gender-based downrating of suicide risk challenge its diagnostic validity? Aust $N Z$ J Psychiatry 2001; 35: 322-8. 